\title{
Modelo e Simulação Numérica de Interações envolvendo Bolhas e Gotas
}

R. MANICA ${ }^{1}$, E. KLASEBOER ${ }^{2}$, Institute of High Performance Computing, 1 Fusionopolis Way, \#16-16 Connexis, Singapore 138632.

D.Y.C. CHAN, ${ }^{3}$ Department of Mathematics and Statistics, The University of Melbourne, VIC 3010, Australia. Faculty of Life and Social Sciences, Swinburne University of Technology, VIC 3122, Australia.

\begin{abstract}
Resumo. Formulamos um modelo teórico para o estudo de escoamento em filmes finos envolvendo superfícies deformáveis (por exemplo bolhas e gotas) que estão interagindo a baixas velocidades. Assumimos que a condição de contorno na interface entre os fluidos é de não-deslizamento tangencial. As equações evolutivas resultantes constituem um sistema algébrico-diferencial na qual a posição da fronteira avança e deforma ao mesmo tempo e depende da solução global do sistema. O foco principal do trabalho é na derivação do modelo e nos detalhes da implementação numérica. As equações são resolvidas usando uma rotina do Matlab e os resultados numéricos são comparados com dados experimentais da literatura que foram produzidos por pesquisadores em diferentes laboratórios e usando diversas técnicas, comprovando que o modelo é adequado para uma variedade de sistemas.
\end{abstract}

Palavras-chave. Sistema algébrico-diferencial, equações de Stokes-Reynolds e Young-Laplace, escoamento em filmes finos, coalescência, gotas e bolhas.

\section{Introdução}

Interações envolvendo bolhas e gotas estão presentes em tarefas do nosso dia a dia como lavar os pratos ou tomar banho, mas também podem ser encontradas em diversos setores indústrias relevantes para a economia brasileira como por exemplo extração de óleo e gás, estabilidade de emulsões, indústria farmacêutica e de cosméticos. Entender a interação entre duas bolhas ou gotas em sistemas multifásicos desempenha um papel fundamental na determinação das características de fluxo de todo o sistema. No entanto, a grande diferença de escalas de comprimento características de tais sistemas implicam em desafios significativos na interpretação dos resultados experimentais e na formulação de modelos teóricos. Por exemplo, para bolhas e gotas com tamanho em torno de $100 \mu \mathrm{m}$, as colisões que podem levar à coalescência são afetadas em parte por forças de superfície (por exemplo Van der

\footnotetext{
${ }^{1}$ manicar@ihpc.a-star.edu.sg; Autor para correspondência

2 evert@ihpc.a-star.edu.sg

${ }^{3}$ D.Chan@unimelb.edu.au
} 
Waals-Lifshitz e elétrica) que operam em escalas de nanômetros. Por isso é importante descrever com precisão as propriedades de fluxo do sistema evolutivo com o mesmo nível de resolução espacial. Além disso, deformações da superfície que são da ordem de nanômetros em bolhas e gotas são também associadas às condições de fluxo e à magnitude das forças de superfície. Fatores como a natureza da condição de contorno hidrodinâmica na superfície das bolhas também contribuem para a sua interação. Finalmente, é importante ter em conta a natureza dinâmica das interações, tais como a dependência das forças de colisão entre bolhas e gotas e determinar se as colisões são estáveis ou levam à coalescência.

O uso de dinâmica de fluidos computacional na qual o sistema global é estudado considerando o movimento das bolhas em um líquido apresenta uma série de dificuldades de identificação da interface e também da necessidade de malhas extremamente finas para capturar detalhes quando as interfaces estão muito próximas. O uso da teoria da lubrificação, na qual somente o filme fino é considerado, permite estudar a fase final do processo de interação e determinar a estabilidade do sistema. A dificuldade na formulação do modelo teórico está no fato de termos um domínio limitado pelo tamanho da bolha na qual definimos a fronteira numa posição fora da zona de interação, mas menor do que seu raio. Essa fronteira avança e deforma simultaneamente e a deformação depende da solução de todo o sistema. Detalhes dessa formulação teórica foram revisados recentemente por Chan et al. [6] bem como comparações com dados experimentais [5]. Soluções analíticas aproximadas para esse sistema só são conhecidas para pequenas deformações [4].

\section{Equações Governantes}

Estamos interessados na interação envolvendo superfícies deformáveis como mostradas na Figura 1. No primeiro exemplo (Figura 1a) vemos uma bolha ancorada a uma seringa, sendo a bolha aproximada de uma superfície plana através do movimento da seringa ou da superfície. Outra possibilidade é injetar ar através da seringa de forma que a bolha cresça e interaja com a superfície. Um experimento como esse foi feito por Fisher et al [9, 10, 11] e a comparação com a teoria desenvolvida nesse trabalho vai ser mostrada na seção de resultados. Já na Figura 2b, temos o caso de duas bolhas ou gotas sendo aproximadas uma da outra devido a uma força exterior, por exemplo a gravidade ou fluxo de cisalhamento. Experimentalmente, as gotas também podem ser aproximadas de maneira mecânica quando uma gota colocada numa superfície sólida plana interage com outra ancorada numa viga como no caso do equipamento chamado 'microscópio de força atômica' [8, 21].

Assumindo que a fase contínua é constituída de um fluido incompressível Newtoniano, o escoamento é modelado pelas equações de Navier-Stokes e pela continuidade escritas na forma $[2,14]$

$$
\begin{aligned}
\rho\left(\frac{\partial \mathbf{u}}{\partial t}+\mathbf{u} \cdot \nabla \mathbf{u}\right) & =-\nabla p+\mu \triangle \mathbf{u} \\
\nabla \cdot \mathbf{u} & =0
\end{aligned}
$$

onde $\rho$ é a massa específica, $\mu$ a viscosidade da fase contínua (assumida constante 

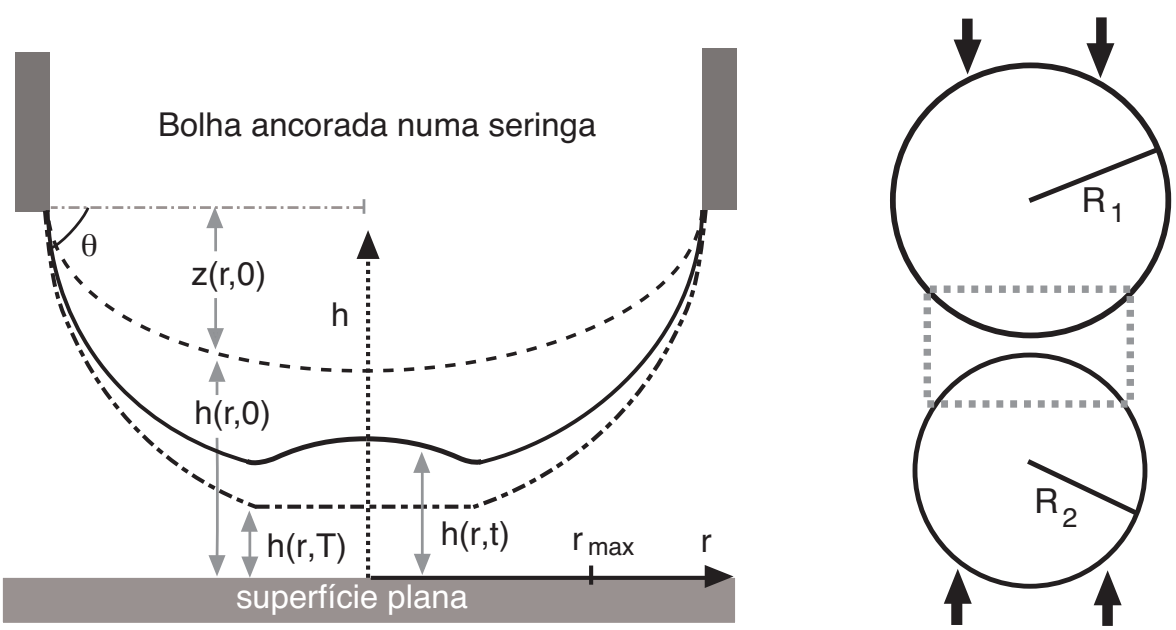

Figura 1: a) Esquema da interação entre uma bolha ancorada numa seringa interagindo com uma superfície plana, onde $z(r, t)$ é a superfície da bolha, enquanto $h(r, 0)$ e $h(r, T)$ representam a separação no tempo inicial 0 e final $T$. b) Interação entre duas bolhas ou gotas com raios $R_{1}$ e $R_{2}$ sendo aproximadas por força exterior.

mesmo para separações muito pequenas na ordem de nanômetros), $p$ é a pressão no filme fino e $\mathbf{u}=(u, v, w)^{T}$ é a velocidade em função do tempo $t$ e de três coordenadas espaciais $(x, y, z)$.

Quando a bolha está próxima da superfície plana ela se deforma de acordo com a Figura 1a. Nesse caso, usando coordenadas polares, as variações em $z$ são muito menores que em $r$. Tomando coordenadas cartesianas $x$ e $y$, isso implica

$$
\frac{\partial h}{\partial x} \ll 1 ; \quad \frac{\partial h}{\partial y} \ll 1
$$

que é uma condição necessária na teoria da lubrificação [19] e no estudo de filmes finos em geral [13]. A validade dessa condição foi discutida nos trabalhos de Chesters $[1,7,20]$. Assumimos também que as velocidades de interação são baixas e consideramos que ocorre fluxo de Stokes na qual o número de Reynolds é pequeno, ou seja, $R e=2 R_{o} \rho V / \mu \ll 1$, onde $R_{o}$ é o raio da bolha não deformada e $V$ a velocidade de aproximação da bolha. Adotamos as escalas sugeridas por Klaseboer et al. [12] que serão definidas no capítulo sobre implementação numérica. Definimos o número capilar $C a=\mu V / \sigma$ como a relação entre forças viscosas e a tensão superficial $\sigma$. Inserindo essas escalas nas equações de Navier-Stokes e continuidade (2.1) e (2.2), assumindo que $C a \ll 1$ e desprezando termos de segunda ordem, resulta nas equações de lubrificação escritas na forma.

$$
\begin{aligned}
& \frac{\partial p}{\partial x}=\mu \frac{\partial^{2} u}{\partial z^{2}} \\
& \frac{\partial p}{\partial y}=\mu \frac{\partial^{2} v}{\partial z^{2}}
\end{aligned}
$$




$$
\begin{aligned}
\frac{\partial p}{\partial z} & =0 \\
\frac{\partial u}{\partial x}+\frac{\partial v}{\partial y}+\frac{\partial w}{\partial z} & =0
\end{aligned}
$$

Considerando a equação (2.5) vemos que $p$ não depende de $z$. Isso permite obter $u$ e $v$ ao integrar as equações (2.3) and (2.4) com relação a $z$ onde assumimos que $u=0$ e $v=0$ nas fronteiras $z=0$ e $z=h$. Note que essa condição de contorno assume não-deslizamento tangencial na interface entre os fluidos, que é contrária à condição usual, mas que está em acordo com os dados experimentais [5]. A bolha está se aproximando da superfície a uma velocidade $w=-d h / d t$, onde $h$ é a distância da bolha à superfície. Substituindo $u, v$ e $w$ na equação (2.6) obtemos a equação de Stokes-Reynolds

$$
\frac{\partial h}{\partial t}=\frac{1}{12 \mu} \frac{\partial}{\partial x}\left(h^{3} \frac{\partial p}{\partial x}\right)+\frac{1}{12 \mu} \frac{\partial}{\partial y}\left(h^{3} \frac{\partial p}{\partial y}\right) .
$$

Note que a equação (2.7) é válida tanto para uma bolha interagindo com uma superfície plana como para duas bolhas interagindo entre si. Para a pressão usamos a equação de Young-Laplace (também conhecida como balanço de tensão normal) escrita na forma

$$
p+\Pi=\frac{2 \sigma}{R}-\frac{\sigma}{n}\left(\frac{\partial^{2} h}{\partial x^{2}}+\frac{\partial^{2} h}{\partial y^{2}}\right),
$$

onde $\Pi(h(r, t))$ é uma força de superfície, por exemplo Van der Waals ou elétrica que depende somente da distância entre as interfaces e $2 \sigma / R$ é a pressão de Laplace. Quando a deformação é pequena, $R \sim R_{o}$ e $2 \sigma / R_{o}$ é a diferença de pressão entre o interior e exterior da bolha. O valor constante $n$ é 1 para uma bolha ou 2 para duas bolhas. Nesse segundo caso, $R_{o}$ é dado pela média harmônica entre $R_{1}$ e $R_{2}$ definido como

$$
\frac{1}{R_{o}}=\frac{1}{2}\left(\frac{1}{R_{1}}+\frac{1}{R_{2}}\right) .
$$

As equações (2.7) e (2.8) são válidas na região de interação quando existe a presença de um filme fino. Precisamos de condições de contorno numa posição $x$ e $y$ fora do filme mas menor que o raio da bolha. Devido a complexidade do sistema bidimensional em encontrar condições de contorno, pois estas variam dependendo do sistema, vamos mostrar resultados assumindo que o sistema é axissimétrico, visto que a maioria dos resultados experimentais apresenta simetria axial.

\section{Equações para o Sistema Axissimétrico}

Assumindo que o sistema mantém simetria axial nos permite resolver o problema em uma única dimensão espacial. Usando o mesmo procedimento do caso bidimensional podemos facilmente chegar às equações [5]

$$
\frac{\partial h}{\partial t}=\frac{1}{12 \mu r} \frac{\partial}{\partial r}\left(r h^{3} \frac{\partial p}{\partial r}\right)
$$




$$
p+\Pi=\frac{2 \sigma}{R}-\frac{\sigma}{n r} \frac{\partial}{\partial r}\left(r \frac{\partial h}{\partial r}\right),
$$

onde $n=1$ para uma bolha e $n=2$ para duas bolhas. Note que se a bolha tem formato esférico isto implica que $p=0$ e no caso de o filme ser plano temos $p=2 \sigma / R$ se assumirmos que a pressão de superfície $\Pi$ é nula. A integral da pressão fornece a força de interação do sistema, que é dada por

$$
F=2 \pi \int_{0}^{\infty} r(p+\Pi) d r
$$

onde $p+\Pi \rightarrow 0$ quando $r \rightarrow \infty$. Para completar o formalismo, precisamos de uma condição inicial e quatro condições de contorno adequadas para o sistema. A condição inicial é dada por

$$
h(r, 0)=h_{0}+\frac{n r^{2}}{2 R_{o}},
$$

onde $h_{0}$ é a separação inicial e assumimos que a superfície da gota ou bolha mantém a sua forma esférica original. As condições de contorno no centro de simetria são dadas por

$$
\begin{aligned}
& \frac{\partial h}{\partial r}=0 \quad \text { em } \quad r=0 \\
& \frac{\partial p}{\partial r}=0 \quad \text { em } \quad r=0 .
\end{aligned}
$$

Escolhemos uma posição $r_{\max }$ (Figura 1a) onde aplicamos as outras duas condições de contorno. Sabemos que fora da área de interação a pressão decai numa função $r^{-4}[22]$ que pode ser implementada como

$$
\frac{\partial p}{\partial r}+\frac{4}{r} p=0 \quad \text { em } \quad r=r_{\max }
$$

Outra possibilidade é simplesmente assumir que a pressão é nula em $r_{\max }$, mas resultados numéricos mostraram que a condição (3.7) fornece resultados mais acurados. Precisamos de uma condição de contorno que forneça o movimento da superfície $\partial h / \partial t$ na posição $r=r_{\max }$. Uma possibilidade é assumir que não existe deformação e que a superfície deformável se aproxima com a velocidade da base sólida $[1,12]$

$$
\frac{\partial h}{\partial t}=\mp V \quad \text { em } \quad r=r_{\max }
$$

onde usamos $-V$ se as superfícies estão se aproximando e $V$ se estão se afastando. Todavia, resultados numéricos [3] mostraram que a condição (3.8) fornece resultados que dependem da escolha de $r_{\max }$, ou seja a solução muda com o tamanho do domínio. Isso se deve ao fato da superfície deformável ter comportamento logarítmico quando uma força exterior é aplicada. A forma assintótica da solução da equação (3.2), assumindo volume constante, é dada por [5]

$$
h(r, t) \sim h(0, t)+\frac{F}{2 \pi \sigma}\left[\ln \left(\frac{F}{8 \pi \sigma R_{o}}\right)+B(\theta)\right],
$$


onde $B(\theta)$ é uma constante que depende do ângulo $\theta$ entre a bolha e a superfície. Esse resultado indica que mesmo uma força pontual tem efeito de deformar toda a bolha, que adequa sua superfície de forma a minimizar energia.

Durante o doutorado do primeiro autor [15], desenvolvemos uma condição de contorno que é independente da escolha de $r_{\max }$ dada por $[3,6]$

$$
\frac{\partial h}{\partial t}+\frac{n}{4 \pi \sigma} \frac{d F}{d t}\left[2+\ln \left(\frac{r_{\max }^{2}}{4 R_{0}^{2}}\right)+\ln \left(\frac{1+\cos \theta}{1-\cos \theta}\right)\right]=\mp V \quad \text { em } \quad r=r_{\max } .
$$

Assim, completamos a formulação teórica do problema. Esse sistema pode ser adaptado para o caso de bolhas ou gotas interagindo sob o efeito da gravidade na qual a força é constante, o que gera um sistema de equações adimensionais totalmente independente de qualquer variável e temos uma solução universal para o problema. Mostraremos a seguir a implementação numérica do sistema evolutivo de equações.

\section{Implementação Numérica}

As equações evolutivas são implementadas na forma adimensional onde usamos as escalas sugeridas por Klaseboer et al. [12] que foram baseadas nas escalas propostas por Chesters [1]. A escala do tempo é dada pela velocidade $t_{c}=h_{c} / V$, a escala da pressão é dada pela pressão de Laplace, a escala radial depende da região onde ocorre deformação $r_{c}^{2}=R_{0} h_{c}$ e a separação $h_{c}$ é escolhida para adimensionalizar as equações. Com essas escolhas as quantidades adimensionais são:

$$
\begin{aligned}
h_{c} & =R_{0} C a^{1 / 2} \\
r_{c} & =R_{0} C a^{1 / 4} \\
p_{c} & =\sigma / R_{0} \\
t_{c} & =\mu C a^{-1 / 2} / p_{c},
\end{aligned}
$$

onde $C a=\mu V / \sigma$ é o número capilar, a relação entre forças viscosas e a tensão superficial. A forma adimensional das equações (3.1), (3.2) e (3.3) fica (usando os mesmos símbolos para as variáveis adimensionais)

$$
\begin{aligned}
\frac{\partial h}{\partial t} & =\frac{1}{12 r} \frac{\partial}{\partial r}\left(r h^{3} \frac{\partial p}{\partial r}\right) \\
p+\Pi & =2-\frac{1}{n r} \frac{\partial}{\partial r}\left(r \frac{\partial h}{\partial r}\right) \\
\frac{F}{2 \pi \sigma}=G & =\int_{0}^{\infty} r[p(r, t)+\Pi(h(r, t))] d r,
\end{aligned}
$$

onde $n=1$ para uma bolha e $n=2$ para duas bolhas. Note que definimos a quantidade $G$ relacionada com a força de interação. As condições iniciais e de contorno ficam

$$
h(r, 0)=h_{0}+\frac{n r^{2}}{2}
$$




$$
\begin{aligned}
& \frac{\partial h}{\partial r}=0 \quad \text { em } \quad r=0 \\
& \frac{\partial p}{\partial r}=0 \quad \text { em } \quad r=0 \\
& \frac{\partial p}{\partial r}+\frac{4}{r} p=0 \quad \text { em } \quad r=r_{\max } \\
& \frac{\partial h}{\partial t}+\frac{n \alpha}{2} \frac{d G}{d t}=\mp 1 \text { em } r=r_{\max },
\end{aligned}
$$

onde

$$
\alpha=\left[2+\ln \left(\frac{r_{\max }^{2} C a^{1 / 2}}{4}\right)+\ln \left(\frac{1+\cos \theta}{1-\cos \theta}\right)\right]
$$

para linha de contato fixa em $r=r_{1}$. Para o caso na qual o ângulo $\theta=\theta_{p}$ é fixo temos [3]

$$
\alpha=\left[2+\ln \left(\frac{r_{\max }^{2} C a^{1 / 2}}{4}\right)+\ln \left(\frac{1+\cos \theta_{p}}{1-\cos \theta_{p}}\right)-\frac{2}{2+\cos \theta_{p}}\right] .
$$

Note que o uso da condição de contorno (4.8) ao invés de (3.8) altera a natureza do problema, pois o sistema se torna algébrico-diferencial, ou seja, a condição de contorno depende da solução do sistema e precisa ser resolvida simultaneamente.

Discretizamos o sistema na coordenada espacial $r$ usando o método da linhas [3] descrito a seguir. Usamos diferenças finitas centrais em $r$ nas equações (4.1) e (4.2) e obtemos um sistema de equações diferenciais ordinárias para $h_{j}(t) \equiv h(j \Delta r, t), j=$ $0 \cdots N$ onde $N=r_{\max } / \Delta r$. Usamos uma malha uniforme $r=\left[0, r_{\max }\right]$ com $\Delta r=$ 0,05 e $r_{\max }=10$ produzindo um sistema de 200 equações. As condições de contorno em $r=0$ são usadas pra obter $\dot{h}_{0}$ e a equação (4.8) fornece a condição para $\dot{h}_{N}$. Isso requer $G$ como uma variável extra a ser resolvida. Ao calcular $G$, dividimos a região de integração em duas partes:

$$
G=\int_{0}^{r_{\max }} r[p+\Pi] d r+\int_{r_{\max }}^{\infty} r p d r
$$

onde a primeira integral é calculada numericamente usando a regra de Simpson. Desta forma, $G$ está relacionada com todas as outras variáveis $h_{j}$ como uma restrição algébrica. A segunda integral, onde $\Pi$ pode ser desprezado, pode ser calculada analiticamente baseado na forma $r^{-4}$ da pressão [22].

Resumindo, o sistema final de equações tem a forma

$$
\left(\begin{array}{ccccc}
1 & 0 & \cdots & 0 & 0 \\
0 & 1 & 0 & \cdots & 0 \\
& & \vdots & & \\
0 & 0 & \cdots & 1 & \alpha \\
0 & 0 & \cdots & 0 & 0
\end{array}\right)\left(\begin{array}{c}
\dot{h}_{0} \\
\dot{h}_{1} \\
\vdots \\
\dot{h}_{N} \\
\dot{G}
\end{array}\right)=\left(\begin{array}{c}
f_{0} \\
f_{1} \\
\vdots \\
\mp 1 \\
G-\sum_{j} \beta_{j} g\left(h_{j}\right)
\end{array}\right),
$$

onde $\beta_{j}$ são os coeficientes da regra de Simpson e os valores $f_{j}$ representam as contribuições da equação de evolução do filme fino. Esse sistema tem matriz massa singular e é algébrico-diferencial de índice 1 e pode ser resolvido usando algum software padrão para EDOs, no nosso caso usando a rotina ode15s no Matlab. 


\section{Exemplos de Aplicações}

Os resultados das simulações numéricas são comparados com dados experimentais da literatura. No primeiro exemplo considerou-se a interação entre uma bolha e uma superfície sólida plana de quartzo na qual dados foram publicados por Fisher et al $[9,10,11]$. Detalhes adicionais sobre esse experimento bem como a teoria implementada podem ser encontrados na literatura recente [5, 17]. No segundo exemplo consideramos a interação entre duas gotas (fase discreta) imersas num segundo líquido (fase contínua), sendo que os resultados experimentais foram obtidos por Klaseboer et al [12]. Durante aquele experimento vários materiais foram usados incluindo água, diferentes tipos de óleo e glicerina. Informações adicionais relacionadas com esse experimento, bem como situações semelhantes de interação entre duas superfícies deformáveis podem ser encontradas em [6, 18].

\subsection{Interação entre uma bolha e uma superfície sólida plana}

O esquema do experimento foi mostrado na Figura 1a na qual uma bolha de ar é injetada de uma seringa que está inicialmente próxima de uma superfície plana. Os resultados experimentais, bem como a solução numérica das equações governantes, são reproduzidos na Figura 2 para um caso de água natural. Os dados experimentais foram extraídos da literatura $[9,10,11]$ onde os autores enfatizaram que nenhum modelo teórico existente era capaz de explicar os resultados obtidos.

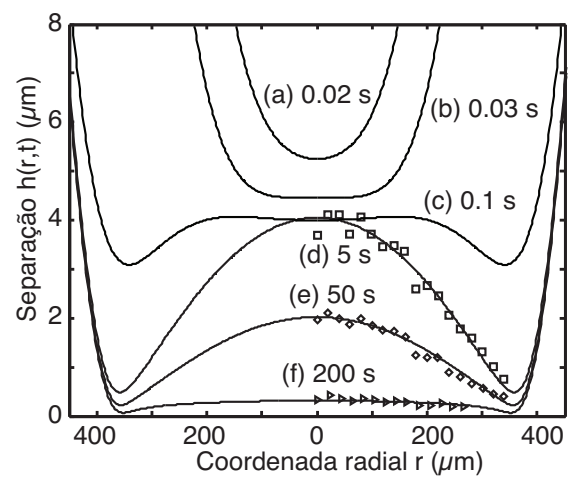

Figura 2: Interação entre uma bolha e uma superfície plana de quartzo em água natural. Linhas contínuas representam o modelo teórico enquanto os símbolos são experimentos que foram obtidos para $t=5,50$ e 200 segundos.

Uma bolha de raio $R_{o} \sim 1.1 \mathrm{~mm}$ está inicialmente a uma distância $h_{0} \sim 40 \mu \mathrm{m}$ da superfície de quartzo. Quando ar é injetado a bolha cresce e eventualmente interage com a superfície. A pressão na parte central de interação se torna superior à sua pressão interna, o que ocasiona uma inversão de curvatura (curvas (a)-(c) na Figura 2). Esse processo inicial ocorre numa escala de tempo rápida e não foi capturado durante o experimento. Durante a fase de escoamento do filme fino (curvas (d)-(f) na Figura 2) o processo é lento durando em torno de 200 segundos, 
onde percebemos uma excelente concordância entre o modelo teórico (linhas sólidas) e o experimento (símbolos) para diferentes tempos. Como o sistema tem simetria axial, o experimento foi conduzido para um único raio. Eventualmente um filme estável aparece devido à repulsão gerada pela força elétrica na superfície (curva (f)). Esse fenômeno também foi observado em experimentos envolvendo gotas de mercúrio interagindo com uma superfície plana [16].

\subsection{Interação entre duas gotas}

Um dos experimentos mais completos que estudam o fluxo e a evolução de filmes finos entre duas gotas iguais (raio $\sim 1.5 \mathrm{~mm}$ ) de mesmo material em um outro líquido foi realizado por Klaseboer et al [12] durante seu trabalho de doutorado. Os dados experimentais foram analisados usando as mesmas equações do presente trabalho, mas a condição de contorno adotada naquele trabalho era dada pela equação (3.8). Embora os autores tenham sido capazes de obter resultados qualitativamente e até quantitativamente precisos, estudos posteriores mostraram que a condição (3.8) não é adequada para este problema [3]. Isso ocorre pelo fato dessa condição de contorno não levar em consideração a deformação das gotas mantendo o volume constante.
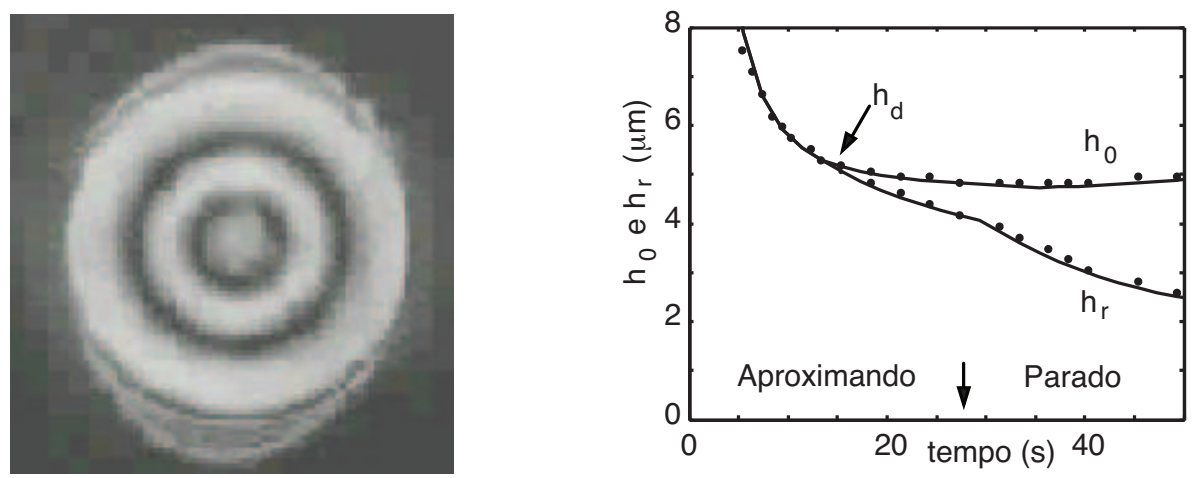

Figura 3: a) Exemplo de anéis de Newton obtidos experimentalmente. b) Evolução da parte central $h_{o}$ e da separação mínima $h_{r}$. As linhas contínuas representam o modelo teórico enquanto os círculos são dados experimentais. A flecha indica o momento que as gotas pararam de ser aproximadas em $t=27 \mathrm{~s}$.

O experimento consiste de duas seringas colocadas uma contra a outra na qual duas gotas foram injetadas nas pontas. Ao aproximar as seringas com velocidade constante $V$, as gotas eventualmente interagem e deformam. O escoamento no filme fino pode ser bem lento se a fase contínua tiver viscosidade alta e demorar muitos minutos e até horas até que ocorra coalescência. Por outro lado, experimentos em água com gotas [8] ou bolhas [21] muito menores $\left(R_{o} \sim 100 \mu \mathrm{m}\right)$ mostraram que a coalescência pode ocorrer em menos de 1 segundo.

Os dados experimentais foram obtidos usando interferômetro onde os anéis de Newton mostrados na Figura 3a podem ser convertidos em separações através da 
relação de Bragg para um anel de ordem $m: h=m \lambda / 2 n$ onde $\lambda=632.8 \mathrm{~nm}$ é o comprimento de onda do laser e $n=1.41$ é o índice de refração do óleo de silicone.

Algumas características importantes do escoamento no filme são a evolução da parte central $h_{0}$ e da posição onde a separação é mínima $h_{r}$, que são mostrados na Figura 3b. Essa posição é onde eventualmente ocorre coalescência desse sistema. Isso ocorre quando $h_{r}$ se torna suficientemente pequeno que a interação de Van de Waals provoca a ruptura do filme. Esse é um exemplo de sistema instável enquanto no exemplo apresentado na seção 5.1 o sistema era estável devido à repulsão elétrica entre a superfície e a bolha.

\section{Conclusão e Perspectivas}

Neste trabalho, apresentamos um sistema de equações algébrico-diferencial que foi derivado baseado em problemas experimentais e nas características físicas da evolução de filmes finos para superfícies deformáveis. Os resultados numéricos foram comparados com dados experimentais da literatura. A excelente concordância mostra que o modelo teórico desenvolvido captura as características físicas dos problemas estudados. O uso de teoria e recursos computacionais proporciona entender diversos fatores que não podem ser facilmente visualizados a partir dos dados experimentais.

Sistemas multifásicos reais encontrados na natureza e em processos indústriais são geralmente mais complicados que os estudados nesse trabalho. A grande maioria das interações não apresenta simetria axial, pode ocorrer coalescência prematura devido a presença de impurezas, efeitos relacionados a números de Reynolds altos e turbulência. Esses efeitos constituem muitas possibilidades para pesquisas futuras.

\section{Agradecimentos}

Gostaríamos de agradecer ao Prof Dr AL De Bortoli (UFRGS) por fornecer inúmeras sugestões que melhoraram consideravelmente o manuscrito. DYC Chan é professor visitante da Universidade Nacional de Cingapura (NUS) e cientista em residência no Instituto do Computação de Alta Performance (IHPC), Cingapura. R Manica gostaria de agradecer a UFRGS por fornecer a base matemática durante graduação e mestrado e a Universidade de Melbourne, Austrália pela bolsa para fazer doutorado pleno sob supervisão de DYC Chan. E finalmente agradece a NUS que forneceu ajuda financeira para fazer intercambio em Cingapura, onde conheceu E Klaseboer e mais tarde se tornou pesquisador no IHPC.

\footnotetext{
Abstract. We developed a theoretical model to study the thin films involving deformable interfaces (for example drops and bubbles) which are interacting at low speeds. We assume the tangentially immobile boundary conditions holds at the fluid-fluid interface. The evolution equations for such system are of differentialalgebraic nature in which the position of the boundary advances and deforms at the same time and the deformation depends on the solution. We focus on the model derivation and numerical implementation. The equations are solved using a Matlab routine and the numerical results are compared to experimental data from the literature, which were produced by researchers in different labs and using different techniques and shows the model is adequate to solve a variety of problems.
} 
Keywords. Differential algebraic system; Stokes-Reynolds equations and YoungLaplace equations; flow in thin films; coalescence, droplets and bubbles.

\section{Referências}

[1] S. Abid, A.K. Chesters, The drainage and rupture of partially-mobile film between colliding drops at constant approach velocity, Int. J. Multiphase Flow, 7 (1994), 613-629.

[2] G.K. Batchelor, "An Introduction to Fluid Dynamics", Cambridge University Press, 1967.

[3] S.L. Carnie, D.Y.C. Chan, C. Lewis, R. Manica, R.R. Dagastine, Measurement of dynamical forces between deformable drops using the atomic force microscope. I. Theory, Langmuir, 21 (2005), 2912-2292.

[4] D.Y.C. Chan, E. Klaseboer, R. Manica, Dynamic deformations and forces in soft matter, Soft Matter, 5 (2009), 2858-2861.

[5] D.Y.C. Chan, E. Klaseboer, R. Manica, Film drainage and coalescence between deformable drops and bubbles, Soft Matter, 7 (2011), 2235-2264.

[6] D.Y.C. Chan, E. Klaseboer, R. Manica, Theory of non-equilibrium force measurements involving deformable drops and bubbles, Adv. Colloid Interface Sci., 165 (2011), 70-90.

[7] A.K. Chesters, Modelling coalescence processes in fluid-liquid dispersions: A review of current understanding, Trans. Inst. Chem. Engrs. Part A, 69 (1991), 259-270.

[8] R.R. Dagastine, R. Manica, S.L. Carnie,D.Y.C. Chan, G.W. Stevens, F. Grieser, Dynamic forces between two deformable oil droplets in water, Science, 313 (2006), 210-213.

[9] L.R. Fisher, E.E. Mitchell, D. Hewitt, J. Ralston, J. Wolfe, The drainage of a thin aqueous film between a solid surface and an approaching gas bubble, Colloids Surf., 52 (1991), 163-174.

[10] L.R. Fisher, D. Hewitt, E.E. Mitchell, J. Ralston, J. Wolfe, The drainage of an aqueous film between a solid plane and an air bubble, Adv. Colloid Interface Sci., 39 (1992), 397-416.

[11] D. Hewitt, D. Fornasiero, J. Ralston, L.R. Fisher, Aqueous film drainage at the quartz/water/air interface, J. Chem. Soc. Faraday Trans., 89 (1993), 817-822.

[12] E. Klaseboer, J.Ph. Chevaillier, C. Gourdon, O. Masbernat, Film drainage between colliding drops at constant approach velocity: experiments and modeling, J. Colloid Interface Sci., 229 (2000), 274-285. 
[13] L.G. Leal, "Laminar Flow and Convective Transport Processes", Butterworth Heinemann, Boston, 1992.

[14] R. Manica, A.L. De Bortoli, Simulations of sudden expansion flows for powerlaw fluids, J. Non-Newtonian Fluid Mech. 121 (2004), 35-40.

[15] R. Manica, "Modelling Hydrodynamic Interactions between Deformable Droplets", PhD Thesis, The University of Melbourne, 2007.

[16] R. Manica, J.N. Connor, S.L. Carnie, R.G. Horn, D.Y.C. Chan, Dynamics of interactions involving deformable drops: hydrodynamic dimpling under attractive and repulsive electrical double layer interactions, Langmuir 23 (2007), 626-637.

[17] R. Manica, D.Y.C. Chan, Drainage of the air-water-quartz film: experiments and theory, Phys. Chem. Chem. Phys., 13 (2011), 1434-1439.

[18] R. Manica, E. Klaseboer, D.Y.C. Chan, Dynamic interactions between drops-a critical assessment, Soft Matter, 4 (2008), 1613-1616.

[19] O. Reynolds, On the theory of lubrication and its application, Philos. Trans. R. Soc. London, A177 (1886), 157.

[20] A. Saboni, C. Gourdon, A. K. Chesters, Drainage and rupture of partially mobile films during coalescence in liquid-liquid systems under a constant interaction force, J. Colloid and Interface Sci. 175 (1995), 27-35.

[21] I.U. Vakarelski, R. Manica, X. Tang, S.J. O’Shea, G.W. Stevens, F. Grieser, R.R. Dagastine, D.Y.C. Chan, Dynamic interactions between microbubbles in water, Proceedings of the National Academy of Sciences of the United States of America, 175 (2010) 11177-11182.

[22] S.G. Yiantsios, R.H. Davis, On the buoyancy-driven motion of a drop towards a rigid surface or a deformable interface, J. Fluid Mech., 217 (1990), 547-573. 